\title{
The compliance coach: A bedside observer, auditor, and educator as part of an infection prevention department's team approach for improving central line care and reducing central line-associated bloodstream infection risk
}

\author{
Mark O. Buchanan BS, BSN, RN, RN-BC a,*, \\ Shelley K. Summerlin-Long MPH, MSW, BSN, RN a, Lauren M. DiBiase MS, CIC ${ }^{\mathrm{a}, \mathrm{b}}$, \\ Emily E. Sickbert-Bennett PhD, MS, CIC ${ }^{\mathrm{a}, \mathrm{b}}$, David J. Weber MD, MPH ${ }^{\mathrm{a}, \mathrm{b}}$ \\ a Department of Hospital Epidemiology, University of North Carolina Hospitals, Chapel Hill, NC \\ ${ }^{\mathrm{b}}$ Division of Infectious Diseases, University of North Carolina School of Medicine, Chapel Hill, NC
}

Key Words:

Continuous quality improvement Health care-associated infections audit

feedback

\begin{abstract}
A compliance coach who audits central line maintenance and provides feedback and education to bedside nurses through timely, nonpunitive conversation is an effective addition to busy infection prevention departments. Staff nurses and nurse managers reported receiving clearly communicated and actionable information from the coach and compliance improved over time in multiple areas of central line maintenance.
\end{abstract}

The role of hospital-based infection preventionists (IPs) has evolved since it was introduced in 1959 in England. ${ }^{1}$ The major difference today is that computerization of medical records combined with high patient to IP ratios has limited and sometimes even eliminated contact between patients and IPs. IPs now rely on bedside staff to report practices that are infection risks, but these practices are often an invisible part of a unit's work culture.

A solution to bridge this gap and help prevent hospital-associated infections (HAIs) is an independent, bedside observer: A clinical compliance coach. A compliance coach serves as a natural extension of infection prevention by directly assessing practice and providing feedback and education in real time to those delivering direct patient care.

\section{METHODS}

This study was conducted at a 933-bed academic medical center. The Infection Prevention Department consists of 4.5 inpatient IPs. During October 2016, a registered nurse experienced in central line

\footnotetext{
* Address correspondence to Mark O. Buchanan, BS, BSN, RN, RN-BC, Department of Hospital Epidemiology, University of North Carolina Hospitals, 1001 West Wing, NCMH, Room W-1063, 101 Manning Dr, Chapel Hill, NC 27514.

E-mail address: mark.buchanan@unchealth.unc.edu (M.O. Buchanan).

Conflicts of interest: None to report.
}

care and nurse precepting was embedded in the department as a compliance coach. The coach began conducting routine, housewide, unannounced audits of central venous access device (CVAD) dressings and intravenous (IV) tubing, using a model of observation, data capture, coaching, and reporting, followed by focused education. Over a 10-month period from November 2016-August 2017 and with internal review board approval, data were collected using visual observation and entered into an online database. Audit frequency on a unit was driven by central line-associated bloodstream infection (CLABSI) rates. Units with high CLABSI rates were audited weekly, whereas units with low rates and few central lines days per year were audited once a quarter.

The compliance coach captured data on dressing condition and date; chlorhexidine gluconate ( $\mathrm{CHG}$ ) sponge placement, including whether or not the line was sutured to allow proper placement; number of IV lines; tubing label compliance (eg, expiration date); and presence of sterile, single-use caps on unattached IV tubing. Audit feedback was given to a patient's assigned nurse or charge nurse. Audit reports were provided to nurse managers who were encouraged to provide staff education on low-compliance areas. Compliance rate data were divided into 3 periods to observe change over time after repeated coach feedback. Compliance rate data were compared with $P$ values.

After 10 months, 2 anonymous online surveys were sent to nurse managers and staff nurses to evaluate acceptance of the compliance 
coach's role and to assess desired frequency of audits, comfort level with unannounced audits, preferences regarding receiving feedback, and clarity and utility of audit information.

\section{RESULTS}

During the 10-month period, the compliance coach observed 626 CVAD dressings and 1,829 IV tubing lines across 31 inpatient units and noted improvement in compliance over time (Fig 1). Two important measures known to prevent CLABSIs are a clean/dry/ intact dressing and a properly placed CHG sponge at the line insertion site. Clean/dry/intact dressing compliance over the period during which the coach was engaged improved from $64 \%$ (160 out of 250 ) in the first evaluation period to $84 \%$ (181 out of 216$)(P=.0001)$ in the final evaluation period. Proper CHG sponge placement improved from $54 \%$ (136 out of 250 ) to $78 \%$ (175 out of 216$)(P=.0001)$. The compliance coach's audit data also showed 121 of 1,829 IV tubing lines of patients with central lines were capped with a nonsterile cap, left open to the air, or looped onto the needleless access port. These shortcuts placed patients at risk for infection from contamination. ${ }^{2}$ Failure to properly cap disconnected IV tubing statistically decreased with focused education from $8.3 \%$ (75 out of 827) during the first evaluation period to $2.6 \%$ (14 out of 514$)(P=.0001)$ during the final evaluation period.

Nineteen of 31 nurse managers (61\%) responded to the anonymous survey evaluating the audits and compliance coach's role, and $100 \%$ approved of unannounced audits. Forty-two percent of respondents preferred quarterly audits and 32\% preferred audits when CLABSI rates were rising. A smaller percentage believed monthly (16\%) or bimonthly (10\%) visits were appropriate. The majority of nurse managers strongly agreed or agreed that the reports were clearly communicated and actionable (Fig 2). Only a few respondents disagreed with these statements.
More than 100 ( $\mathrm{n}=106$ ) bedside nurses responded to the survey. Approximately one-half (47\%) preferred to receive feedback outside of patient rooms. About one-quarter (27\%) of those surveyed wanted feedback at the bedside. A smaller group of respondents preferred the data be shared with the charge nurse (16\%), e-mailed to the nurse placing the dressing (4\%), or desired no feedback (6\%).

Overall, $21 \%$ of bedside nurse respondents (22 out of 106) indicated they interacted with the compliance coach (Fig 2). These nurses overwhelmingly (95\%) believed the coach communicated clearly about patients' CVAD care and $85 \%$ stated the information was actionable.

\section{DISCUSSION}

Many factors contributed to improved CVAD dressing and IV labeling compliance, but having a compliance coach on the units provided essential infection prevention data we would not have had access to without someone at the bedside. The coach was able to capture unit CVAD dressing compliance over time and across units while providing one-on-one central line care education. Feedback provided at the bedside or outside patient rooms requires a compliance coach with strong communication skills because the effectiveness of audits and feedback depends on how feedback is provided. ${ }^{3}$ Gaps in knowledge and practice were reported to nursing leadership and IPs so targeted education could occur at the unit level. Persistent improvement in catheter care is associated with providing feedback to nurses and nurse managers. ${ }^{4}$

Bedside nurses are key members of the CLABSI prevention team, but they have multiple competing priorities. Unit staff may overlook practices that are an infection risk because they become part of their daily routine. A trained outside observer can be key to identifying poor infection prevention practices. Survey results indicate that the role of compliance coach was widely accepted by nursing

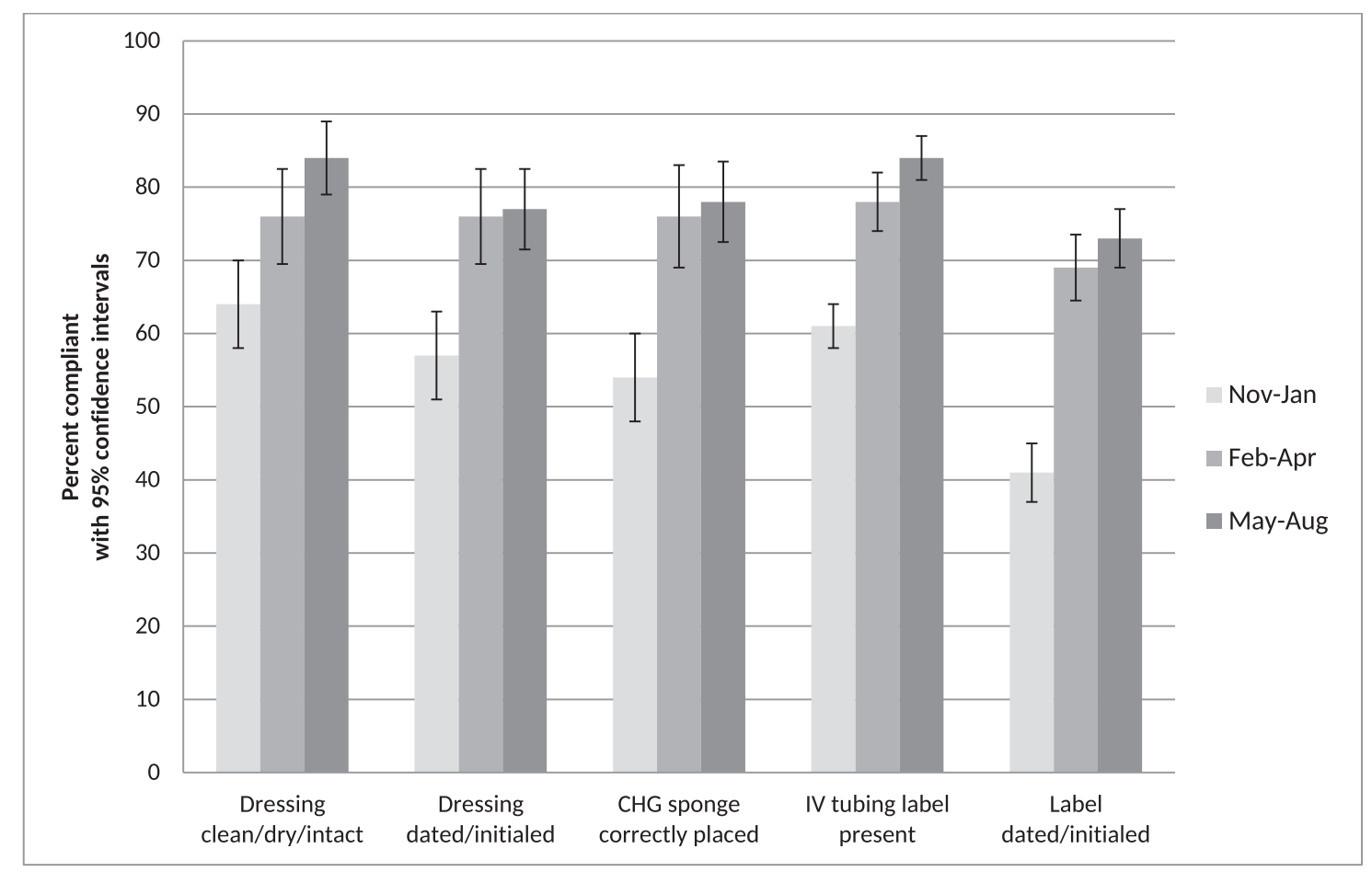

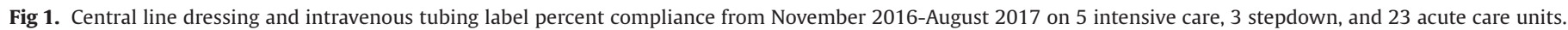
$C H G$, chlorhexidine gluconate; $I V$, intravenous line. 


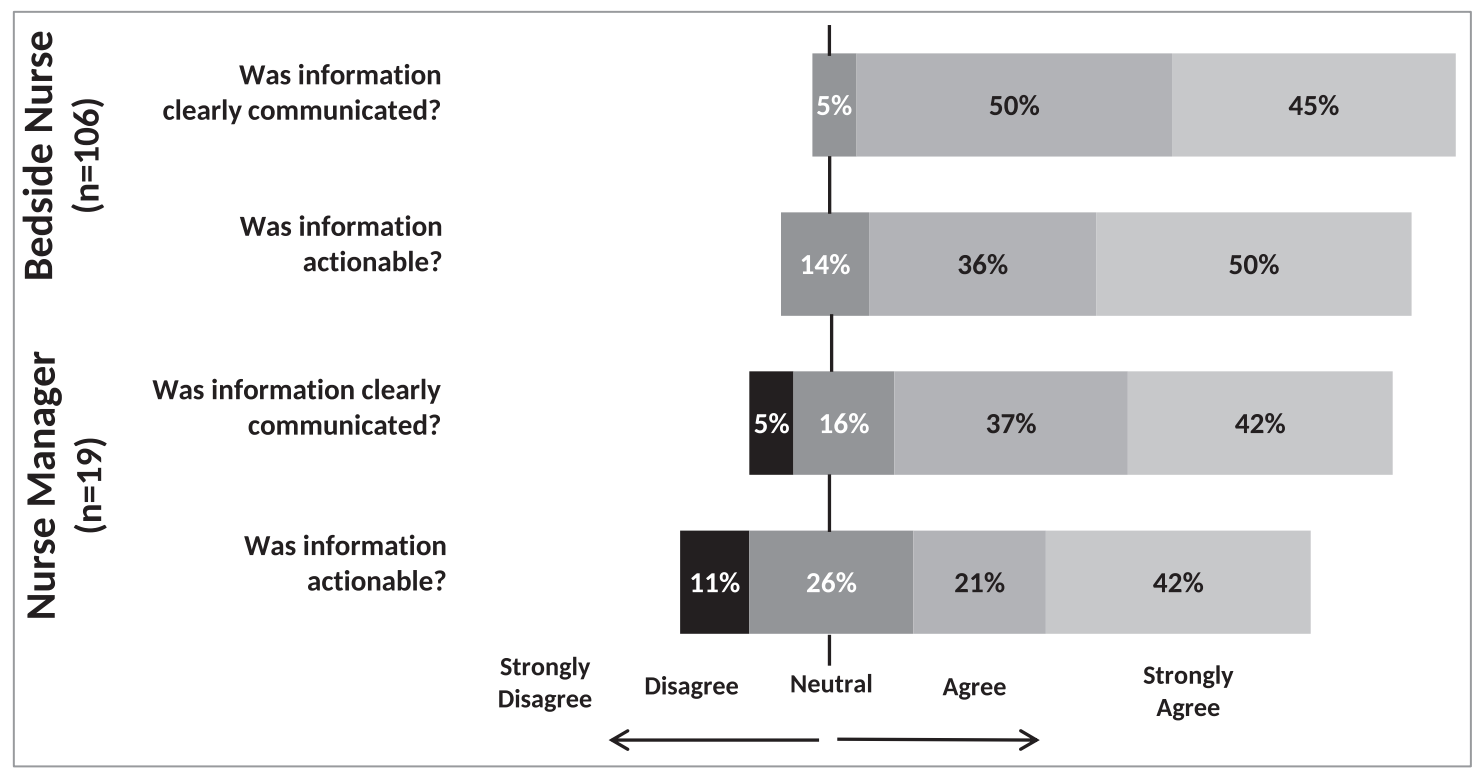

Fig 2. Value of the compliance coach's one-on-one interaction with feedback.

staff. Although this study focused on process measures and did not directly assess changes in CLABSI rates, units that received the most audits, feedback, and education did observe decreased CLABSI rates during the evaluation period, which was likely attributable in part to the compliance coach. Our study has some limitations. Units were not selected randomly for CVAD observations, but based on their higher CLABSI rates and central line days. Feedback was provided to staff for the purpose of improving performance so the Hawthorne effect in a sense was our intervention. Our survey response rate for nurse managers was reasonable (61\%) and we had more than 100 bedside nurses respond. It is possible that their responses are not representative of all nurse managers and bedside nurses invited to respond.

\section{CONCLUSIONS}

In today's high-cost health care environment, the economic reality is that patients, insurance companies, and government are no longer willing to pay to treat preventable infections. IPs have only limited time to be at bedsides. HAI prevention requires new strategies and partnerships. Having dedicated quality improvement personnel such as a compliance coach embedded in a busy infection prevention department can be a valuable addition. Although the importance of audits and feedback is well known, ${ }^{5,6}$ what is unique is a position in infection prevention that takes time-limited initiatives and makes them ongoing. Having a compliance coach at the bedside interacting with the front-line team is a useful tool in HAI prevention.

\section{Acknowledgments}

The authors thank the following infection preventionists for providing unit education and support: Lisa Teal, BSN, RN, CIC; Katherine Schultz, MPH, BSN, RN; Cynthia Culbreth, BSN, RN, RN-BC; Christa Clark, BSN, RN, CCRN; and Brooke Brewer, MS, BSN, RN, CNML. The authors also thank Gail Burdette, BSN, RN, for sharing her technical knowledge related to central venous access device care in pediatric populations.

\section{References}

1. Gardner A, Stamp M, Bowgen J, Moore B. The infection control sister: a new member of the control of infection team in general hospitals. Lancet 1962;280:710-1. doi:10.1016/s0140-6736(62)90520-2.

2. Grissinger M. Capping intravenous tubing and disinfecting intravenous ports reduce risks of infection. P T 2011;36:62-76.

3. Ivers N, Jamtvedt G, Flottorp S, Young JM, Odgaard-Jensen J, French SD, et al. Audit and feedback: effects on professional practice and healthcare outcomes. Cochrane Database Syst Rev 2012;(6):CD000259. doi:10.1002/14651858.cd000259.pub3.

4. Fakih MG, Jones K, Rey JE, Berriel-Cass D, Kalinicheva T, Szpunar S, et al. Sustained improvements in peripheral venous catheter care in non-intensive care units: a quasi-experimental controlled study of education and feedback. Infect Control Hosp Epidemiol 2012;33:449-55. doi:10.1086/665322.

5. Fakih MG, Jones K, Rey JE, Takla R, Szpunar S, Brown K, et al. Peripheral venous catheter care in the emergency department: education and feedback lead to marked improvements. Am J Infect Control 2013;41:531-6. doi:10.1016/ j.ajic.2012.07.010

6. Cherry MG, Brown JM, Neal T, Ben Shaw N. What features of educational interventions lead to competence in aseptic insertion and maintenance of CV catheters in acute care? BEME Guide No. 15. Med Teach 2010;32:198-218. doi:10.3109/01421591003596600. 